Anforderungen der Unternehmen an den Verkehr

\title{
Die Stadt als Gewerbestandort
}

\author{
Die Zukunft von Handel und produzierendem Gewerbe in der Stadt wird im \\ Licht der sich verändernden ökonomischen Bedinungen und vor dem Hinter- \\ grund räumlicher Auflösungsprozesse zunehmend kritisch. Der Logistik eines \\ Unternehmens kommt eine zentrale Rolle für den Unternehmenserfolg zu. \\ Wie können die Ansprüche der Unternehmen mit denen einer ökologisch ver- \\ träglichen Mobilitäł vereint werden?
}

$\mathrm{T}$

Von Markus Hesse echnische und wirtschaftliche Wandlungsprozesse besitzen erhebliche Folgen für den Verkehrssektor, die Stadtplanung und ökologie. Welchen Einfluß haben veränderte ökonomische Rahmenbedingungen und unternehmerische Anpassungsstrategien auf die Verkehrsentwicklung und damit auf urbane Ökosysteme und wie stark sind die Handlungsspielräume der Planung davon bestimmt? Drei Betrachtungs- und Handlungsebenen gilt es zu untersuchen und zu berücksichtigen:

- externe, regionsseitig nicht veränderbare Rahmenbedingungen der Wirtschafts- und Verkehrsentwicklung,

- die Gesamtheit regionaler „Settings“, aus denen sich Standortqualitäten und Standortwirkungen ergeben,

- individuelle Handlungsmuster vor allem von Unternehmen, die über ihre Verkehrsnachfrage und Flächennutzung direkt ökologisch wirksam werden.

Die Zukunft von Handel und produzierendem Gewerbe in der Stadt ist stark abhängig von den ökonomischen Verhältnissen des Umfelds und von den gegebenen räumlichen Strukturen. Beides ist in den letzten Jahren starken Veränderungen unterworfen und wirkt sich teilweise kritisch auf die Situation der Unternehmen aus. Die Kommunen wiederum haben Probleme in ihrer Zielfindung, da diese (beispielsweise urbane Mischungen contra Gewerbeverbund) sich teilweise widersprechen. Der Befund aus den Beispielstädten (Bremen, Halle u.a.) bestätigt, daß die Erfolgsbedingungen für eine umweltorientierte Verkehrsplanung sehr stark über den Umgang mit ökonomischen Interessen und Zielkonflikten definiert werden (eine Aussage, die sicher auch auf andere Städte übertragbar ist). Allerdings muß der Strukturwandel nicht zwangs- läufig in der Verkehrsspirale enden, es gibt auch gegenläufige, ökologisch effiziente Tendenzen.

\section{- Veränderte Umfeldfaktoren}

Veränderte ökonomische Rahmenbedingungen haben das Umfeld der Unternehmen und damit auch ihre Verkehrsansprïche erheblich beeinflußt. Die wichtigsten Faktoren sind:

- Ausweitung und Internationalisierung der Märkte und eine zunehmend distanzintensive räumlich-funktionale Arbeitsteilung (Globalisierungseffekt);

- neue Materialfluß- und Transportketten sowie entsprechend feinregulierte, sensible Zeitregimes im Rahmen flexibler Produktionskonzepte (Logistikeffekt);

- Rückgang der Grundstoffe und Massengüter bei wachsender Bedeutung von Investitionsgütern und Konsumgütern (Güterstruktureffekt);

- neue Konzepte der logistischen Leistungserstellung mit neuen Profilen in der Transportwirtschaft (unter anderem als Folge zunehmender Netzwerkorganisation);

- Liberalisierung und Deregulierung von Märkten, vor allem die Aufhebung von Marktzugangsbeschränkungen und Wettbewerbsordnungen im Verkehrsgewerbe.

Dieser umfassende Strukturwandel hat vor dem Hintergrund der unterschiedlichen Potentiale der einzelnen Verkehrsträger zu einer Steigerung der insgesamt transportierten Mengen geführt und die Entwicklung des Straßengüterverkehrs begünstigt, der die neuen Anforderungen mit seinen systemeigenen Vorteilen (hohe Netzbildung, kurzfristige Flexibilität, geringe Transaktionskosten) bisher offenbar besser erfüllen konnte als die Bahn (hohe Laufzeiten bei hohen relativen Preisen, zum Teil keine
Orts- bzw. Marktpräsenz, Angebotslücken bei Teilladungen, wenig produktspezifische Angebote) oder das Binnenschiff.

\section{Anpassungsstrategien und Standortansprïche der Betriebe}

Auf der lokalen Ebene äußern sich die Anpassungsstrategien der Betriebe sehr verschieden: $\mathrm{Zu}$ einem Anwachsen der Verkehrserzeugung und Transportnachfrage kommt es vor allem im Rahmen der heute häufig angestrebten ,just in Time"-Produktionsweise aufgrund der Substitutionseffekte zwischen Warenbestand (fast weitgehender Abbau der kapitalintensiven Lagerhaltung) und Warenbewegung (kurzfristige Disposition von Produktions- und Lieferrhythmen). Hieraus ergeben sich nach allen bisherigen Erfahrungen höhere spezifische Transportintensitäten pro Betrieb oder belegter Fläche. Diese Konsequenz zeigt sich in unterschiedlicher Form sowohl bei der Industrie als auch im Handel.

Die verstärkt übliche ,fließende“ Fertigung in eingeschossigen Hallen beansprucht größere Flächen als der traditionelle Stockwerksbau. Auch wird der Abbau von Lagerflächen in den Produktionsbetrieben (die in aller Regel als

\section{Hauptsachen}

Christian Laesser

Verkehrs- und Umweltproblematik in städtischen Gebieten Analyse, Lösungsmöglichkeiten, Auswirkungen. Untersucht am Beispiel der Stadt und Verkehrsregion St. Gallen

Der motorisierte Individualverkehr hat sich in den vergangenen Jahren stürmisch entwickelt. In zunehmendem Masse treten jedoch negative Auswirkungen dieses Verkehrswachstums in Erscheinung. Verschiedene gegensteuernde Massnahmen werden diskutiert. Doch über die Art und das Ausmass der Auswirkungen solcher Eingriffe bestehen bis anhin grosse Unsicherheiten und deshalb Meinungsunterschiede.

Dieses Buch nimmt eine äusserst detaillierte und tiefgreifende Analyse möglicher Auswirkungen verkehrslenkender Massnahmen vor. Einige praktische Fallbeispiele für die Stadt und Verkehrsregion St. Gallen geben einen vertieften und illustrierten Einblick in die eigens zu diesem Zweck entwickelte Methode.

1996. 562 Seiten, 170 Abb., 21 Tab., DM 87.- ISBN 3-258-05337-5

\section{Verlag Paul Haupt} Falkenplatz $14 \cdot \mathrm{CH}-3001$ Bern Fax $0041 \cdot 31 \cdot 3014669$ 
Reserven gebunden sind und somit nicht in den Naturhaushalt zurückgefiuhrt werden können) durch Flächennachfrage in der Distributionswirtschaft überkompensiert. Dieser Effekt äußert sich in der räumlichen Expansion transportintensiver Nutzungen, etwa der Branche Transport/Umschlag/Lagerei. In der Region Halle-Leipzig hat sich beispielsweise das Verkehrsgewerbe bereits mehr oder minder spontan an der Peripherie nahe der Autobahn A 14 angesiedelt. Dort laufen Planungen zur Zusammenlegung von Nutzungen und zur Bündelung von Transporten über ein großes Güterverkehrszentrum (GVZ) auch marktseitig ins Leere. Am Beispiel des GVZ Bremen läßt sich hingegen studieren, wohin die städtebauliche und ökologische Überdimensionierung der Logistik führen kann. Solche „Dinosaurier" lassen sich in kaum einer anderen Stadt realisieren beziehungsweise sinnvoll betreiben.

Insofern sind beide Städte dem Drahtseilakt ausgesetzt, daß einerseits wirtschaftliche Tätigkeiten unbedingt gefördert werden müssen, andererseits hat aber die bisher stattindende motorisierte Mobilität bereits das Maß dessen, was man als zuträglich bezeichnen könnte, überschritten. Die bisher praktizierte Strategie, gewerbliche Nutzungen vorrangig mit Blick auf stadtökonomische Ziele und Interessen zu planen beziehungweise deren Bestand zu sichern, sollte daher zumindest um eine vorsorgende, planerische Integration des Wirtschaftsverkehrs ergänzt werden. Entsprechende Handlungsmöglichkeiten beschränken sich gleichwohl nicht auf die ressortbezogene Planung, sondern sollten interdisziplinär zwischen Städtebau, Verkehr und Wirtschaftsressort verfolgt werden. Denn bisher werden solche Potentiale aufgrund fehlender Querbezüge zwischen der Stadtentwicklungsplanung (soweit vorhanden), Verkehrsplanung und
Wirtschaftsförderung in der Praxis - wenn uiberhaupt - nur eher zufällig ausgeschöpft.

\section{Zukünfte und Modernisierungsstrategien}

Soll der Verkehr ökologisch orientiert gestaltet werden, so setzt dies eine offensive Auseinandersetzung mit den Ansprüchen der Unternehmen voraus. Es zeigen sich Handlungsspielräume auf, die mit den Dispositionen der Betriebe in Übereinstimmung gebracht werden können (z.B. Unternehmenslogistik). Ein solches Reduktionsmodell wäre aber an eine problembewußte, zeitgemäße Kommunikationsstrategie gebunden, die sich nicht in der Polarisierung konkurrierender Interessen und Nutzungsansprüche erschöpft, sondern nach strategischen Schnittstellen zwischen einzelwirtschaftlichen und gesamtstädtischen Zielen sucht (z.B. Stadtlogistik). Die bisher favorisierten verkehrlichen Wachstumsmodell basieren hingegen auf der Annahme, daß der ökonomische Wohlstand einer Stadtregion per se auf Motorisierung und Mobilität angewiesen ist und eine derartige wachsende Verkehrsnachfrage auch gesättigt werden muß.

Sollten diese klassischen Ansätze durchbrochen werden, so müssen Verkehrs- und Stadtentwicklungsplaner - jenseits einer solchen oberflächlichen Annahme - nach den Schnittstellen zwischen privatem und öffentlichem Interesse suchen und diese für eine praktische Umsetzung nutzen. Auf der Ebene der regionalen „Settings“ wie auch der direkten Steuerung gehören dazu unter anderen:

- Aufbau einer bestands- und qualitätsorientierten Strategie der Standortsicherung für das verarbeitende Gewerbe;

- in Verbindung damit Ausschöpfung von Synergieeffekten zwischen Verladern, Transportgewerbe und Logistikstandorten für einen stadtverträglichen Wirtschaftsverkehr;
- Entwicklung der Informations- und Kommunikationstechnik zu einem tatsächlichen Steuerungspotential für Transportströme und Verkehrsabläufe (Logistik).

Auf längere Sicht sind zudem interessante Tendenzen im Strukturwandel auszumachen, die jenseits der Logik von den Sachzwängen neue Handlungsoptionen eröffnen:

- die tendenzielle Entkoppelung der Wertschöpfung von der materiellen Produktion;

- die Wiederaneignung des Raumes und die Wiederentdeckung der Region als wichtige Voraussetzung für strukturelle Verkehrsvermeidung.

Aufbauend auf diese Ergebnisse wird das Forschungsprojekt des BMBF ,ökologisch verträgliche Mobilität in Stadtregionen" der Blickwinkel in der weiteren Arbeit des Projektes in Abstimmung mit den Städten auf folgende Schwerpunkte gelegt: Logistik (insbesondere Distribution), Produktionsfunktionen (Gewerbegebiete; Bauwirtschaft) sowie Standortfragen des Handels.

\section{Literafur}

- D. Henckel (Hrsg.): Produzierendes Gewerbe in der Stadt. Dokumentation eines Workshops im Rahmen des Projekts "Entscheidungsfelder städtischer Zukunft". DifuMaterialien 11/95, Berlin 1995

- Industrie- und Handelskammer zu Berlin: Entwicklungsstrategien für Industriestandorte in der Region Berlin-Brandenburg. Von der Flächensicherung zur Standortqualifizierung, Berlin 1995

- B. Lutz, M. Hartmann, H. Hirsch-Kreiensen (Hrsg.): Produzieren im 21. Jahrhundert. Herausforderungen für die deutsche Industrie. Ergebnisse des Expertenkreises „Zukunftsstrategien" Band I, Frankfurt 1996

Der Autor
Markus Hesse, Dipl.Geogr, Leiter des Forschungs-
feldes, "Stadtentwicklung, Planung, Verkehr" des
IÖW, Berlin

Bewertung des Strukturwandels und Planungskonsequenzen

\begin{tabular}{|c|c|c|}
\hline Tendenzen in Richtung Verkehrswachstum & Tendenzen in Richtung Verkehrseinsparung & Konsequenzen för die Planung \\
\hline $\begin{array}{l}\text { Dezentroler, selbstorganisierter Ablouf von Marktprozessen } \\
\text { (0.Steverung) }\end{array}$ & $\begin{array}{l}\text { Steverungspotential der Logistik, } \\
\text { zur Transportoptimierung und -vermeidung }\end{array}$ & $\begin{array}{l}\text { Integierte Standortplanung zwischen Flachennuizung } \\
\text { und Verkehr }\end{array}$ \\
\hline $\begin{array}{l}\text { Durhsetzungsmacht der logistischen Rationolisierung } \\
\text { als regionsextern geselzes Roum-/Zeir-Modell }\end{array}$ & $\begin{array}{l}\text { Evolution van, „new industrial districts" als } \\
\text { rummiche Re-Konzentration der Standorte (cluster) }\end{array}$ & $\begin{array}{l}\text { Förderung sinnvoller räumlicher Vernetzungen } \\
\text { von Betrieben in Gewerbeparks und Gemengelagen } \\
\text { (Strofe, Schiene, Schnittstellen) }\end{array}$ \\
\hline $\begin{array}{l}\text { Trend des Industriesystems in Richtung "Größen- } \\
\text { wachstum" (Konzentration, "Globalisierung") }\end{array}$ & $\begin{array}{l}\text { Trend des Industriesystems zur Nähre, u.a. durch das } \\
\text { Miniaturisierungspotential neuer Technologien }\end{array}$ & $\begin{array}{l}\text { Innovationsstrategien mit lokalen und regionalen Pro- } \\
\text { blemlösungen verbinden }\end{array}$ \\
\hline $\begin{array}{l}\text { Zunehmende (Zwangs-) Mobilität van Materie, Personen } \\
\text { und Informationen in "spaces of flow" }\end{array}$ & $\begin{array}{l}\text { Schliebung von Stoffkreisläufen auf regioncler tbene } \\
\text { (etwa durch regionales Stoffstrommanagement) }\end{array}$ & $\begin{array}{l}\text { Vernetzungen zwischen Anbietern und Nachfragern } \\
\text { entlang regionaler Wertschöptungsketten (z.B. regio- } \\
\text { nale Zulieferbörsen) }\end{array}$ \\
\hline
\end{tabular}


(c) 20I0 Authors; licensee IÖW and oekom verlag. This is an article distributed under the terms of the Creative Commons Attribution Non-Commercial No Derivates License (http://creativecommons.org/licenses/by-nc-nd/3.o/), which permits unrestricted use, distribution, and reproduction in any medium, provided the original work is properly cited. 